University of Nebraska - Lincoln

DigitalCommons@University of Nebraska - Lincoln

Ralph Skomski Publications

Research Papers in Physics and Astronomy

May 2002

\title{
Exact nucleation modes in arrays of magnetic particles
}

\author{
Ralph Skomski \\ University of Nebraska-Lincoln, rskomski2@unl.edu
}

Follow this and additional works at: https://digitalcommons.unl.edu/physicsskomski

Part of the Physics Commons

Skomski, Ralph, "Exact nucleation modes in arrays of magnetic particles" (2002). Ralph Skomski Publications. 17.

https://digitalcommons.unl.edu/physicsskomski/17

This Article is brought to you for free and open access by the Research Papers in Physics and Astronomy at DigitalCommons@University of Nebraska - Lincoln. It has been accepted for inclusion in Ralph Skomski Publications by an authorized administrator of DigitalCommons@University of Nebraska - Lincoln. 


\title{
Exact nucleation modes in arrays of magnetic particles
}

\author{
R. Skomskia) \\ Department of Physics and Astronomy and Center for Materials Research and Analysis, \\ University of Nebraska, Lincoln, Nebraska 68588
}

\begin{abstract}
Magnetization reversal in arrays of magnetic nanoparticles with perpendicular anisotropy is investigated. Aside from domain-wall propagation effects, the reversal involves two main aspects: the nucleation behavior of individual particles and interparticle interactions. Due to magnetostatic self-interaction effects, the interparticle interaction cannot be reduced to a stray-field correction to the external field. Exact nucleation fields and explicit stray-field and self-interaction contributions are obtained for rings of equidistant dots. An exact treatment of self-interactions in various structurally inhomogeneous but rotationally symmetric wire, sphere, and thin-film nanostructures leads to renormalization of the uniaxial anisotropy. Finally, an approximate method to calculate nucleation fields is discussed. (C) 2002 American Institute of Physics. [DOI: 10.1063/1.1452251]
\end{abstract}

\section{INTRODUCTION}

The search for rigorous solutions in micromagnetism has been a challenge for many decades. ${ }^{1,2}$ One reason is the involvement of Maxwell's equations, which are difficult to treat due to their nonlocal character. Some exact solutions are the curling and coherent-rotation modes in perfect ellipsoids of revolution ${ }^{3}$ and the quasicoherent bulging and clamped curling modes in nanostructures. ${ }^{4}$ The problem of a very elongated prolate spheroid remained highly controversial for many years, but quite recently it was proven that no third mode (buckling) exists. ${ }^{5}$

This work focuses on particle arrays with perpendicular anisotropy. The structures, which have attracted much attention in several areas of magnetism, are of considerable technological interest, for example, as storage media, sensors, and permanent magnets. ${ }^{6,7}$ In this article we present some exact results, discuss their ranges of applicability, and elucidate the relation between exact and approximate solutions. Some of the results are also of interest for systems with in-plane anisotropy, such as multilayers. ${ }^{8,9}$

\section{MICROMAGNETIC FREE ENERGY}

Since micromagnetic processes are realized by rotations of the magnetization vector, it is convenient to consider the local magnetization direction $\mathbf{s}(\mathbf{r})=\mathbf{M}(\mathbf{r}) / M_{s}$. In terms of the $\mathbf{s}$, the relevant micromagnetic free energy is

$$
\begin{aligned}
F= & \int\left(A(\nabla \mathbf{s})^{2}-K_{1}(\mathbf{r}) s_{z}^{2}-\left(\mu_{0} / 2\right) M_{s} \mathbf{H}_{d}(\mathbf{s}) \mathbf{s}\right. \\
& \left.-\mu_{0} M_{s} \mathbf{H s}\right) d V,
\end{aligned}
$$

where $A$ is the exchange stiffness, $K_{1}$ is the first uniaxial anisotropy constant, $H_{d}$ is the magnetostatic self-interaction field, and $H$ is the applied field (external field). For simplicity, we assume that $A$ is constant throughout the magnet. Inhomogeneous or discontinuous exchange interactions

\footnotetext{
${ }^{a)}$ Electronic mail: rskomski@unlserve.unl.edu
}

$A(\mathbf{r})$, for example, at grain boundaries, amount to modified boundary conditions ${ }^{7,10}$ but are of secondary importance in the present context.

The nucleation field $H_{N}$ investigated in this work is the field $\mathbf{H}=-H_{N} \mathbf{e}_{z}$ at which the remanent magnetization state becomes unstable. When this state is close to perfect alignment, as is assumed in this work, we can write

$$
\mathbf{s}(\mathbf{r})=\mathbf{e}_{z}\left(1-\frac{1}{2} m(\mathbf{r})^{2}\right)+\mathbf{m}(\mathbf{r}),
$$

where $\mathbf{m}=m_{x} \mathbf{e}_{x}+m_{y} \mathbf{e}_{y}$ is a small perpendicular magnetization component. Putting Eq. (2) into Eq. (1) yields

$$
\begin{aligned}
F= & \int\left(A(\nabla \mathbf{m})^{2}-K_{1}(\mathbf{r}) \mathbf{m}^{2}-\frac{1}{2} \mu_{0} M_{s} H_{z} \mathbf{m}^{2}\right) d V \\
& +F_{m s}(\mathbf{m}),
\end{aligned}
$$

where $F_{m s}$ is the magnetostatic self-interaction contribution. To discuss nucleation it is necessary to consider all terms linear and quadratic in the small quantity $\mathbf{m}$; eigenmode analysis of the resulting free energy then yields the soughtfor nucleation field.

The most difficult part of Eq. (3) to treat is the magnetostatic self-interaction term, which can also be written as

$$
F_{m s}=\frac{\mu_{0} M_{s}^{2}}{8 \pi} \int \frac{\nabla \mathbf{s}(\mathbf{r}) \nabla \mathbf{s}\left(\mathbf{r}^{\prime}\right)}{\left|\mathbf{r}-\mathbf{r}^{\prime}\right|} d V d V^{\prime} .
$$

Substituting Eq. (2) into Eq. (4) yields a number of surface and bulk terms with well-defined physical meanings. Aside from terms associated with bulk magnetization inhomogeneities, there are two main contributions. First, the $z$ component of the local field interacts with $s_{z}$. As we can see from Eq. (2), this demagnetizing-field contribution yields a term proportional to $\mathbf{m}(\mathbf{r})^{2}$. Second, there is a self-interaction associated with the perpendicular magnetization component $\mathbf{m}$. This contribution is an integral over $\mathbf{m}(\mathbf{r}) \mathbf{m}\left(\mathbf{r}^{\prime}\right)$ or, alternatively, over $\nabla \mathbf{m}(\mathbf{r}) \nabla \mathbf{m}\left(\mathbf{r}^{\prime}\right)$. For example, from the definition of the curling mode, ${ }^{3}$

$$
\mathbf{m}(r)=m_{0}(r, z) \sin \phi \mathbf{e}_{x}-m_{0}(r, z) \cos \phi \mathbf{e}_{y},
$$




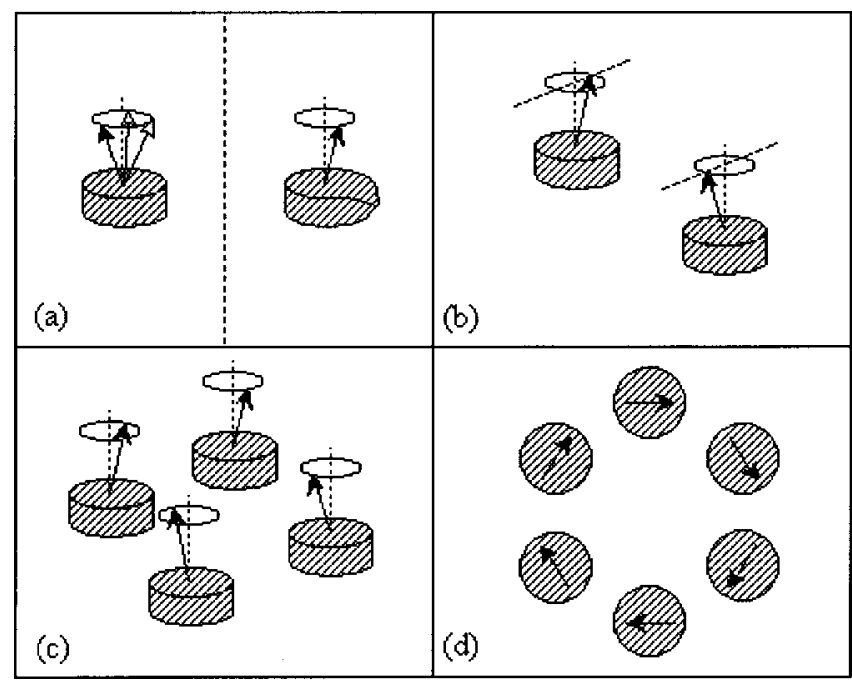

FIG. 1. Schematic single-particle and many-particle nucleation modes: (a) single particle, (b) two particles, (c) particles on a square, and (d) ring of $N=6$ particles.

it follows that $\nabla \mathbf{m}(\mathbf{r})=0$. By contrast, coherent rotation creates surface charges, because $\mathbf{m}$ has a component perpendicular to the surface.

\section{ARRAY INTERACTIONS}

The distinction between external interaction and selfinteraction fields carries over to particle arrays. Figure 1 shows rings consisting of $N$ particles (dots) with perpendicular anisotropy. We assume that the particles, of volume $V$, are ellipsoidal and sufficiently small, so that the local field is homogeneous throughout each particle. Using Eq. (2), we can express the magnetization directions $\mathbf{s}_{i}(i=1, \ldots, N)$ in terms of perpendicular magnetization components $\mathbf{m}_{i}$. Neighboring particles create a magnetostatic interaction field which adds to the external field, but this is not the only consideration, because flux closure associated with $\mathbf{m}_{i}$ reduces the magnetostatic self-interaction energy. Examples of this flux closure are shown in Figs. 1(c) and 1(d).

In the case of a ring of $N$ equidistant dots, the nucleation problem has an analytic solution. Figures 1(c) and 1(d) show the geometry for $N=4$ and 6 , respectively. The nucleation mode is a macroscopic generalization of the curling mode. The result of the calculation is the nucleation field,

$$
H_{N}=\frac{2 K_{1}}{\mu_{0} M_{s}}+\frac{1}{2}\left(1-3 D_{\mathrm{dot}}\right) M_{s}-\frac{M_{s} V}{4 \pi d^{3}}\left(c_{z}+c^{*}\right),
$$

where $d$ is the nearest-neighbor center-to-center distance. The first two terms in this equation are the magnetocrystalline and shape-anisotropy fields of the dots, whereas the last term describes the interactions between the dots. The $c_{z}$ contribution is the interaction field created by neighboring dots, whereas the $c^{*}$ term reflects the self-interaction of the perpendicular magnetization components $\mathbf{m}_{i}$. Figure 2 compares $c_{z}$ and $c^{*}$ for rings of various size. In the limit $N$ $=\infty$, where the ring degenerates into a linear chain, $c_{z}$ $=2.404$ and $c^{*}=2 c_{z}$. In the case of a dumbbell, $N=2$,

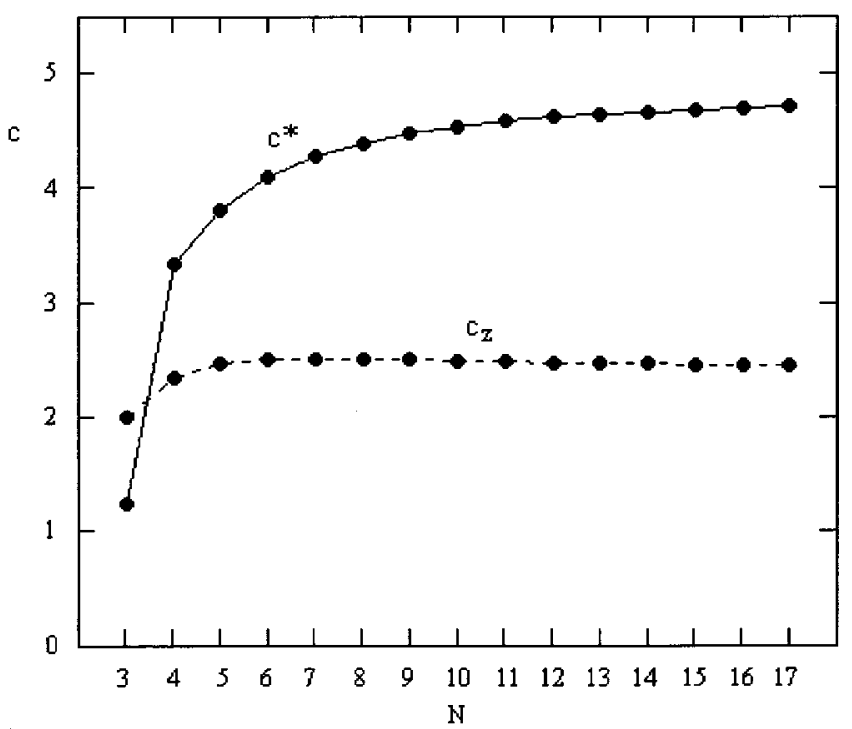

FIG. 2. Demagnetizing-field contributions (dashed lines) and selfinteraction contributions (solid lines) to the nucleation-field of ring structures with perpendicular anisotropy.

nucleation is actually realized by coherent rotation along the dumbbell axis, so that Fig. 1(b) shows an excited mode.

\section{SINGLE-PARTICLE MODES}

In sufficiently small particles (e.g., very thin wires, small spherical particles, ultrathin film patches) the nucleation mode is coherent, that is, $\mathbf{m}(\mathbf{r})=$ const. In a narrower sense, coherent rotation is limited to structurally homogeneous ellipsoids of revolution, but in some cases it is possible to define quasicoherent modes, characterized by $d \mathbf{m} / d \phi=0 .{ }^{11}$ Figure 3 shows some geometries in which there are welldefined quasicoherent modes. For the cases shown in Fig. 3, the magnetostatic energy can be incorporated into $K_{1}$, giving rise to an effective anisotropy constant of $K_{\text {eff }}$. The solution of the nonlocal nucleation problem then reduces to finding the eigenfunctions of an ordinary differential equation. In the cases in Figs. 3(b) and 3(c), the eigenfunctions are superpo-

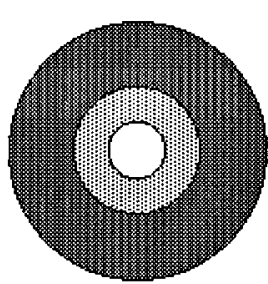

(a) (b)

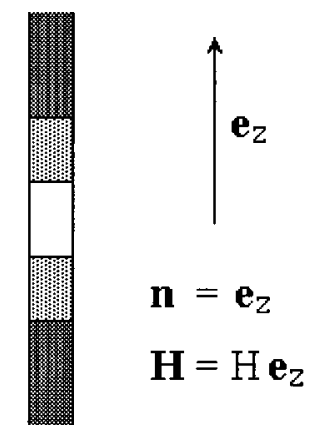


sitions of exponential functions, ${ }^{3,7}$ whereas the modes corresponding to Fig. 3(a) are spherical Bessel functions., ${ }^{3,11}$

For spherical symmetry, $F_{m s}$ is constant, that is, independent of $\mathbf{m}(r)$. One way of showing this is to divide a magnet of the type in Fig. 1(a) into shells of thickness $4 \pi r^{2} d r$ : the shells do not interact because the field inside a homogeneously magnetized shell is zero. ${ }^{11,12}$ Simular arguments apply to other ellipsoids of revolution, such as coated cylinders (not shown in Fig. 3), but then the individual shells or "tubes" have an m-dependent self-energy.

In the limit of thin wires, the surface charges amount to an effective anisotropy constant $K_{\text {eff }}(z)=K_{1}(z)+\mu_{0} M_{s}^{2} / 4$ (see, e.g., Ref. 13). In thin films, Fig. 3(c), we exploit that $\nabla \mathbf{B}$ $=0$ at the interface between any adjacent layers and obtain $\mathbf{H}_{d}=-M_{z}(z) \mathbf{e}_{z}$. (For homogeneous thin films, this mechanism was discussed in Ref. 4). For the considered wire, sphere, and thin-film geometries, these findings can be summarized in terms of the

$$
K_{\text {eff }}(\mathbf{r})=\mathbf{K}_{1}(\mathbf{r})+\frac{1}{4} \mu_{0} M_{s}^{2}\left(1-3 D_{g}\right),
$$

where $D_{g}$ is equal to $0,1 / 3$, and 1 , respectively.

\section{APPROXIMATE SOLUTIONS}

The exact solutions discussed up to now are useful to study idealized systems, but the importance of defects was recognized long ago. ${ }^{2}$ Here we consider the case where magnetization reversal starts at the end of a wire. To investigate the influence of the wire ends we exploit a quantummechanical analogy ${ }^{7}$ and apply Ritz's variational method. In quantum mechanics, the ground-state energy is obtained by minimizing $E=\langle\psi|H| \psi\rangle /\langle\psi \mid \psi\rangle$. In the micromagnetic case, the nucleation field is obtained by minimizing the field expression,

$H_{N}=\frac{2}{\mu_{0} M_{g}} \frac{\int\left(A(\nabla \mathbf{m})^{2}+K_{\mathrm{eff}} \mathbf{m}^{2}+\frac{1}{2} \mu_{0} M_{s} \Delta H(\mathbf{r}) \mathbf{m}^{2}\right) d V}{\int \mathbf{m}^{2} d V}$.

As in quantum mechanics, the method yields comparatively accurate eigenvalues (nucleation fields), even if the trial mode is only moderately realistic.

As a trial function we use $\mathbf{m}(\mathbf{r})=m_{0} \quad \mathbf{e}_{x} \exp \left[-\left(z_{0}\right.\right.$ $-z) / \lambda]$, where $z_{0}$ denotes the position of the wire end and $\lambda$ is the inverse localization length ${ }^{13}$ of the mode. Since the field is not known exactly, we approximate it by $\Delta H=$ $-M_{s}$ for $z_{0}-R<z<z_{0}$ and zero elsewhere. Equation (8) then yields

$$
H_{N}=\frac{2}{\mu_{0} M_{s}}\left[A \lambda^{2}+K_{\mathrm{eff}} \frac{1}{2} \mu_{0} M_{s}^{2}[1-\exp (-\lambda R)]\right] .
$$

Minimizing this expression with respect to $\lambda$ and substituting $\lambda$ into Eq. (9) yields the corrected nucleation field. In thin wires, $\lambda \approx 0$ and $H_{N}=2 K_{\text {eff }} / \mu_{0} M_{s}$. Finite-thickness corrections involve the dimensionless parameter $\mu_{0} M_{s}^{2} R^{2} / A$ and reduce $H_{N}$. In "soft-magnetic" wires, this correction is essentially comparable to the uncorrected nucleation field, and an empirical rule is that the coecivity is about one third of the theoretical nucleation field. However, this popular rule does not apply to hard-magnetic wires, where $K_{1} \gg \mu_{0} M_{s}^{2}$ and the relative nucleation-field reduction is much lower. (Defects in hard magnets often cause deviations from the prediction $2 K_{1} / \mu_{0} M_{s},{ }^{12,14}$ but this is a different mechanism.)

\section{DISCUSSION AND CONCLUSIONS}

A key assumption in Sec. III was that all dots are equivalent. In reality, this equivalence is broken due to geometrical nonequivalence or to dot imperfections. In the general case of $n$ nonequivalent small dots, the interaction problem reduces to diagonalization of a $2 n \times 2 n$ matrix. For small imperfections, the nucleation field may still calculated analytically, but very complicated structures require numerical methods ${ }^{15}$ that go beyond the scope of this work.

The nonequivalence of the dots becomes important when the associated change in the single-dot nucleation field is comparable to or larger than the interaction field. The behavior of the array then changes from cooperative, as in Fig. 1(d), to noncooperative (single particle like). It is important to note that the mechanisms discussed in this work remain operational at finite temperatures, because the energy barriers responsible for coercivity are much larger than $k_{B} T$. As a consequence, modes with more negative instability fields, $H_{z}<-H_{N}$, are difficult to excite thermally and therefore irrelevant to the nucleation problem.

In conclusion, we have discussed exact solutions of nucleation problems for particles and particle arrays with perpendicular anisotropy. In rings of equidistant dots, interactions between identical particles lead to cooperative effects. The nucleation field contains both demagnetizing field and self-interaction contributions and can be calculated exactly. Exact solutions have also been found for the magnetostatic self-energy of some inhomogeneous nanostructures.

\section{ACKNOWLEDGMENTS}

This work was supported by DOE, DARPA/ARO, and AFOSR.

${ }^{1}$ W. F. Brown, Micromagnetics (Wiley, New York 1963).

${ }^{2}$ A. Aharoni, Rev. Mod. Phys. 34, 227 (1962).

${ }^{3}$ A. Aharoni, Introduction to the Theory of Ferromagnetism (Oxford University Press, Oxford, 1996).

${ }^{4}$ R. Skomski, H.-P. Oepen, and J. Kirschner, Phys. Rev. B 58, 3223 (1998).

${ }^{5}$ A. Aharoni, J. Phys.: Condens. Matter 9, 10009 (1997).

${ }^{6}$ D. J. Sellmyer, M. Yu, R. A. Thomas, Y. Liu, and R. D. Kirby, Phys. Low-Dimens. Struct. 1-2, 155 (1998).

${ }^{7}$ R. Skomski and J. M. D. Coey, Phys. Rev. B 48, 15812 (1993).

${ }^{8}$ M. Sawicki, G. J. Bowden, P. A. J. de Groot, B. D. Rainford, J. M. L. Beaujour, R. C. C. Ward, and M. R. Wells, Phys. Rev. B 62, 5817 (2000).

${ }^{9}$ S. Nieber and H. Kronmüller, Phys. Status Solidi B 153, 367 (1989).

${ }^{10}$ R. Skomski, in Spin Electronics, edited by M. Ziese and M. J. Thornton (Springer, Berlin, 2001), p. 204.

${ }^{11}$ R. Skomski, J. P. Liu, and D. J. Sellmyer, Phys. Rev. B 60, 7359 (1999).

${ }^{12}$ R. Skomski and J. M. D. Coey, Permanent Magnetism (Institute of Physics, Bristol, 1999).

${ }^{13}$ R. Skomski, H. Zeng, M. Zheng, and D. J. Sellmyer, Phys. Rev. B 62, 3900 (2000).

${ }^{14}$ H. Kronmüller, Phys. Status Solidi B 144, 385 (1987).

${ }^{15}$ T. Schrefl, J. Fidler, and H. Kronmüller, Phys. Rev. B 49, 6100 (1994). 